\title{
Circulating Exosomal miR-17 Inhibits the Induction of Regulatory T Cells via Suppressing TGFBR II Expression in Rheumatoid Arthritis
}

\author{
Liping Wang $^{a} \quad$ Chunyan Wang ${ }^{a} \quad$ Xuqiang Jia ${ }^{b} \quad$ Jing Yuc \\ aDepartment of Rheumatology and Immunology, Lanzhou University Second Hospital, Lanzhou, \\ bDepartment of Rheumatology and Endocrine, Dingxi People's Hospital, Dingxi, 'Department of \\ Cardiology, Lanzhou University Second Hospital, Lanzhou, China
}

\section{Key Words}

Exosome $\bullet$ miR-17 • Treg cells $\bullet$ TGFBR II Rheumatoid arthritis

\begin{abstract}
Background/Aims: A reduced prevalence of circulating regulatory T cells (Tregs)is a hallmark of inflammatory rheumatoid arthritis (RA). However, the underlying mechanisms of alterations of Tregs are unclear. Methods: The ratio of Tregs in peripheral blood of healthy controls (HCs) and patients with RA was determined by flow cytometry. MicroRNA (miRNA) expression profiles in exosomes derived from RA patients (RA-exosomes) and in those from HCs (HCexosomes) were detected by microarray analysis, and miR-17 was measured by quantitative real-time PCR. Transforming growth factor beta receptor II (TGFBR II) expressed by T cells was measured by flow cytometry. The interaction between miR-17 and TGFBR II was evaluated by dual-luciferase reporter assay. Results: We found that RA-exosomes can selectively affect Treg differentiation in vitro. Several miRNAs are more abundant in the RA-exosomes than in $\mathrm{HC}$-exosomes. Among those upregulated in patients with RA, miR-17 can suppress Treg induction by inhibiting the expression of TGFBR II. Conclusion: Our findings imply that altered miRNA expression in RA-exosomes may contribute to the pathogenesis of RA by disrupting the homeostasis of Tregs.

\section{Introduction}

Rheumatoid arthritis (RA) is the most common type of autoimmune disease. It is characterized by synovial inflammation, production of autoantibodies such as anticitrullinated protein antibody $(\mathrm{Ab})$, and destruction of cartilage and bone. The current understanding of RA pathogenesis is that this equilibrium is disturbed, in part due to qualitative defects of regulatory $\mathrm{T}$ cells (Tregs) [1]. Lawson et al. observed a reduced

\begin{tabular}{ll}
\hline Jing Yu & Department of Cardiology, Lanzhou University Second Hospital \\
& Lanzhou, 730030 (China) \\
& E-Mail yujing2304@126.com
\end{tabular}

\section{KARGER}




\section{Cellular Physiology Cell Physiol Biochem 2018;50:1754-1763 \begin{tabular}{ll|l} 
and Biochemistry & $\begin{array}{l}\text { DOI: 10.1159/000494793 } \\
\text { Published online: 2 November } 2018\end{array}$ & $\begin{array}{l}\text { ( } 2018 \text { The Author(s). Published by S. Karger AG, Basel } \\
\text { www.karger.com/cpb }\end{array}$ \\
\hline
\end{tabular}}

prevalence of circulating $\mathrm{CD} 4{ }^{+} \mathrm{CD} 25^{\text {high }}$ Tregs in patients with early active RA[2]. Ehrenstein et al. reported a defect of Tregs in the suppression of pro-inflammatory cytokine production [3], and Nie et al. showed a reduced suppression of proliferating effector cells by Tregs that was TNF-dependent [4]. However, the underlying mechanisms of alterations of Tregs are unclear.

MicroRNAs (miRNAs) are small ( 22-nucleotide-long) endogenous noncoding RNAs that function as negative regulators of gene expression through complimentary antisense binding to their target mRNAs [5-7]. Alterations of miRNAs in the circulation, inflammatory cell populations, or pathological samples of autoimmune diseases have also been documented [8-11]. miRNA scan be present in exosomes, which are extracellular vesicles smaller than 150 $\mathrm{nm}$ in diameter. Exosomes can affect the target cells via gene regulation, which is mediated by transfer of miRNAs [12-14]. Critical involvement of exosomes has been demonstrated in various human disorders, including cancer and neurodegenerative diseases $[15,16]$. However, exosomal miRNA function in Treg differentiation has not been studied in RA.

In this study, we isolated circulating exosomes from the blood of patients with RA (RAexosomes) and evaluated the potential pathogenic function of these miRNA-containing exosomes in Treg differentiation. We found that RA-exosomes can selectively affect Tregs in vitro. Several miRNAs are more abundant in RA-exosomes than in exosomes from healthy control donors (HC-exosomes). Among those upregulated in patients with RA, miR-17 can suppress Treg induction by inhibiting the expression of transforming growth factor beta receptor II (TGFBR II). Our findings imply that altered miRNA expression in RA-exosomes may contribute to the pathogenesis of RA by disrupting the homeostasis of Tregs.

\section{Materials and Methods}

\section{Human subjects}

We present demographics of the patients with RA and healthy volunteers who agreed to provide peripheral blood for this study in Table 1. The 2010 ACR/EULAR criteria were used to diagnose RA. None of the patients had received therapy. The Ethics Committee of Lanzhou University Second Hospital approved the study protocol.

\section{Isolation of exosomes}

Exosomes were purified from the plasma according to the manufacturer's instructions (Cell Guidance Systems, Cambridge, UK). Briefly, plasma samples were subjected to sequential centrifugation at $1200 \times g$ for $15 \mathrm{~min}$ and at $2000 \times \mathrm{g}$ for $20 \mathrm{~min}$, and supernatant was collected after the final centrifugation at 10, $000 \times g$ for $45 \mathrm{~min}$. The resulting supernatant was mixed with a one-fifth volume of polyethylene glycol buffer (30\% PEG 6000, $50 \mathrm{mmol} / \mathrm{L} \mathrm{HEPES,} 1 \mathrm{~mol} / \mathrm{L} \mathrm{NaCl}$ ). Exosomes were pelleted by centrifugation at 10, $000 \times g$ for $30 \mathrm{~min}$ and resuspended in phosphate-buffered saline (PBS). The size distribution and purity of the collected exosomes was determined using a NanoSight LM10 nanoparticle analysis system (NanoSight Ltd, Malvern, UK). More than $95 \%$ of the extracellular vesicles' size was smaller 200 $\mathrm{nm}$, and the purity of the collected exosomes was higher than $95 \%$.

\section{$C D 4^{+} T$ cell isolation}

Peripheral blood mononuclear cells (PBMCs) were isolated from healthy control (HC) donor blood samples by Ficoll centrifugation (Axis-Shield Diagnostics, Dundee, UK).CD4+T cells (greater than $95 \% \mathrm{CD} 4^{+} \mathrm{T}$ cells) were purified from PBMCs using a MACS CD4+T cell Isolation Kit (Miltenyi Biotec, Bergisch Gladbach, Germany).

Table 1. Clinicopathological characteristics of patients $(\mathrm{n}=25)$

\begin{tabular}{ll}
\hline Variables & No. and (\%) \\
\hline No. of patients & 25 \\
Age (median; range), years & $60.2 ; 29-78$ \\
Sex (male/female) & $5 / 20(20.0 / 80.0)$ \\
RF (positive/negative) & $22 / 3(88.0 / 12.0)$ \\
CRP (mean;range), (mg/dL) & $2.8 ; 0.3-10.4$ \\
Krenn's score & \\
4 & $2(8.0)$ \\
5 & $2(8.0)$ \\
6 & $4(16.0)$ \\
7 & $8(32.0)$ \\
8 & $5(20.0)$ \\
9 & $4(16.0)$ \\
\hline
\end{tabular}


RNA isolation and quantitative real-time PCR

Total RNA from exosomes was isolated using TRIzol reagent (Takara, Dalian, China). Before performing quantitative RT-PCR assays (qPCR), RNA was reverse transcribed into miRNA cDNA and total cDNA using a One-Step PrimeScript miRNA cDNA Synthesis Kit (Takara) and PrimeScript RT reagent Kit (Takara), respectively. The mRNA and miRNA expression levels were detected by qPCR with an Applied Biosystems 7500 Fast Real-Time PCR System (Applied Biosystems, Foster City, CA) with SYBR Premix Ex Taq (Takara) according to the manufacturer's instructions, and were normalized versus GAPDH mRNA and small nuclear RNA U6, respectively. The Ct value of miR-17, miR-19b, miR-121, and TGFBR II was quantified using the 2$\Delta \Delta$ Ct method.

\section{Cell culture}

To determine the roles of exosomes and miRNAs, CD4+ $\mathrm{T}$ cells were cultured with exosomes before or after being transfected with miRNA mimics, inhibitor, and small interfering RNAs (siRNAs) as indicated. RPMI-1640 (Life Technologies, Carlsbad, CA) was used for the culture. The exosomes were derived from the same amount of the plasma and the $\mathrm{CD} 4^{+} \mathrm{T}$ cells were prepared from HCs. Culture cells were stimulated with $1 \mu \mathrm{g} / \mathrm{mL}$ anti-CD3 monoclonal Ab (mAb) (OKT3) and $1 \mu \mathrm{g} / \mathrm{mL}$ anti-CD28 mAb (Beckman Coulter, Brea, CA) for $1 \mathrm{~h}$ at $37^{\circ} \mathrm{C}$ before cells were added. In the differentiation assay, CD4+ $\mathrm{T}$ cells were polarized towards Tregs with $10 \mathrm{ng} / \mathrm{mL}$ TGF- $\beta$ and $100 \mathrm{U} / \mathrm{mL}$ IL2 (PeproTech, Rocky Hill, NJ). To stain intracellular cytokines, cultured cells were stimulated with $30 \mathrm{ng} / \mathrm{mL}$ phorbol-myristate-acetate (Sigma-Aldrich, St. Louis, MO), $200 \mathrm{ng} / \mathrm{mL}$ ionomycin (Sigma-Aldrich), and $1 \mu \mathrm{M}$ monensin (Sigma-Aldrich) for $4 \mathrm{~h}$ before staining.

\section{Flow cytometry}

The Abs used for flow cytometry were FITC-conjugated mouse anti-human CD4, PE-conjugated mouse anti-human Foxp3, APC-conjugated mouse anti-human TGFBR II, and PECY-Cy5.5-conjugated mouse antihuman CD25 from BD Biosciences (San Jose, CA). The cultured cells were collected, washed twice, and resuspended in $100 \mu \mathrm{L}$ of PBS containing $0.1 \%$ bovine serum albumin (BSA). These cells were stained and labeled with either specific Abs or the appropriate isotype controls. The cells were incubated on ice for 30 min, washed with PBS containing $0.1 \% \mathrm{NaN}_{3}$ and $0.5 \% \mathrm{BSA}$, and then fixed in 1\% paraformaldehyde solution. Analyses were performed using FACScan and CellQuest software (BD Biosciences).

\section{Oligonucleotide transfection}

Transfected miR-17, miR-19b, miR-121 mimic, miR-17 inhibitor, negative control (NC) duplex, and siRNA against TGFBR II (siTGFBR II), were all synthesized by GenePharma (Shanghai, China). Oligonucleotide transfection was performed using Lipofectamine 2000 (Invitrogen, Carlsbad, CA) according to the manufacturer's protocol. The transfection rate was approximately $90 \%$.

\section{Statistical analysis}

Results are expressed as the mean \pm standard error of the mean (SEM) and statistically evaluated by use of the two-tailed unpaired $t$ test. A $P$ value less than 0.05 was considered statistically significant. Statistical analyses were performed by GraphPad Prism 5.0 (GraphPad Software, Inc., La Jolla, CA).

\section{Results}

Treg frequency is decreased in RA patients and inhibited by RA-exosomes

Given the critical contribution of cellular immunity in RA patients, we analyzed the ratio of Tregs in peripheral blood of HCs and patients with RA by flow cytometry. As shown in Fig. $1 \mathrm{~A}$ and $\mathrm{B}$, Treg frequency was significantly decreased in RA patients compared with HCs.

To investigate the function of the circulating exosomes in RA patients, we purified exosomes from the plasma of HCs and RA patients. The average size of the vesicles purified from the plasma samples was $89.5 \mathrm{~nm}(n=10)$. The size of most of the purified vesicles was consistent with that of exosomes, which are smaller than $150 \mathrm{~nm}$ (Fig. 1C). Furthermore, they expressed the conventional exosome markers CD9 and CD63 (Fig. 1D). 


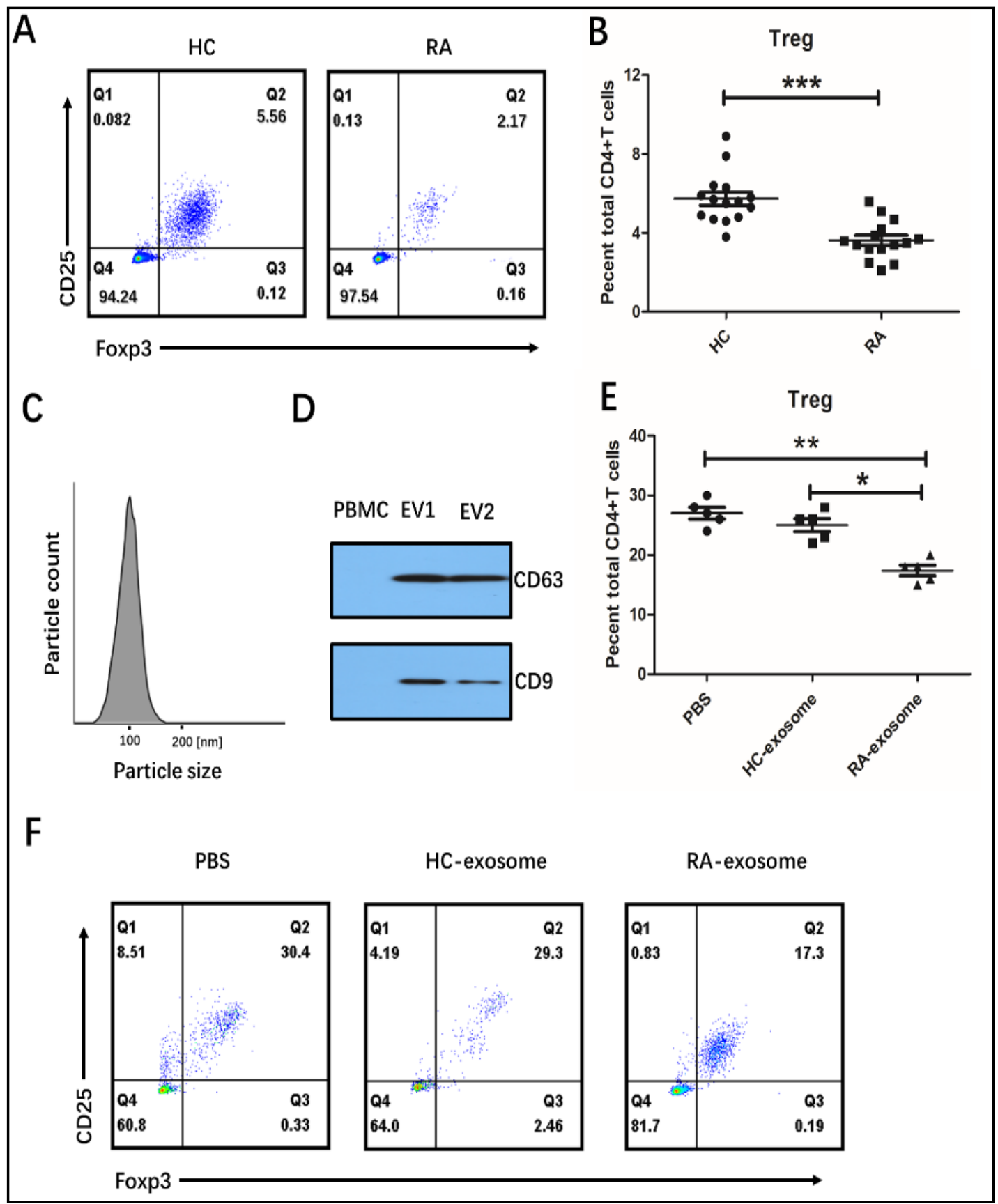

Fig. 1. Treg frequency is decreased in RA patients and inhibited by RA-exosomes. A and B, Dot plot and histogram of flow cytometry data. A, Representative Treg frequency of HC and RA patients. B, The data indicate the mean \pm SEM of 13 subjects. C, Representative size distribution of purified exosomes. Using a NanoSight LM10 nanoparticle analysis system, the size was analyzed three times for each sample. D, Western blot analysis for CD9 and CD63. The PBMC and exosome samples (EV1 and EV2) were collected from RA patients. Each lane was loaded with $5 \mu \mathrm{g}$ of protein for blotting. E and F, Frequencies of Tregs among CD4 ${ }^{+}$ $\mathrm{T}$ cells after culture with exosomes derived from $\mathrm{HC}$ and RA patients. F, One representative experiment is shown. E, Data indicate the mean \pm SEM of three independent experiments. 


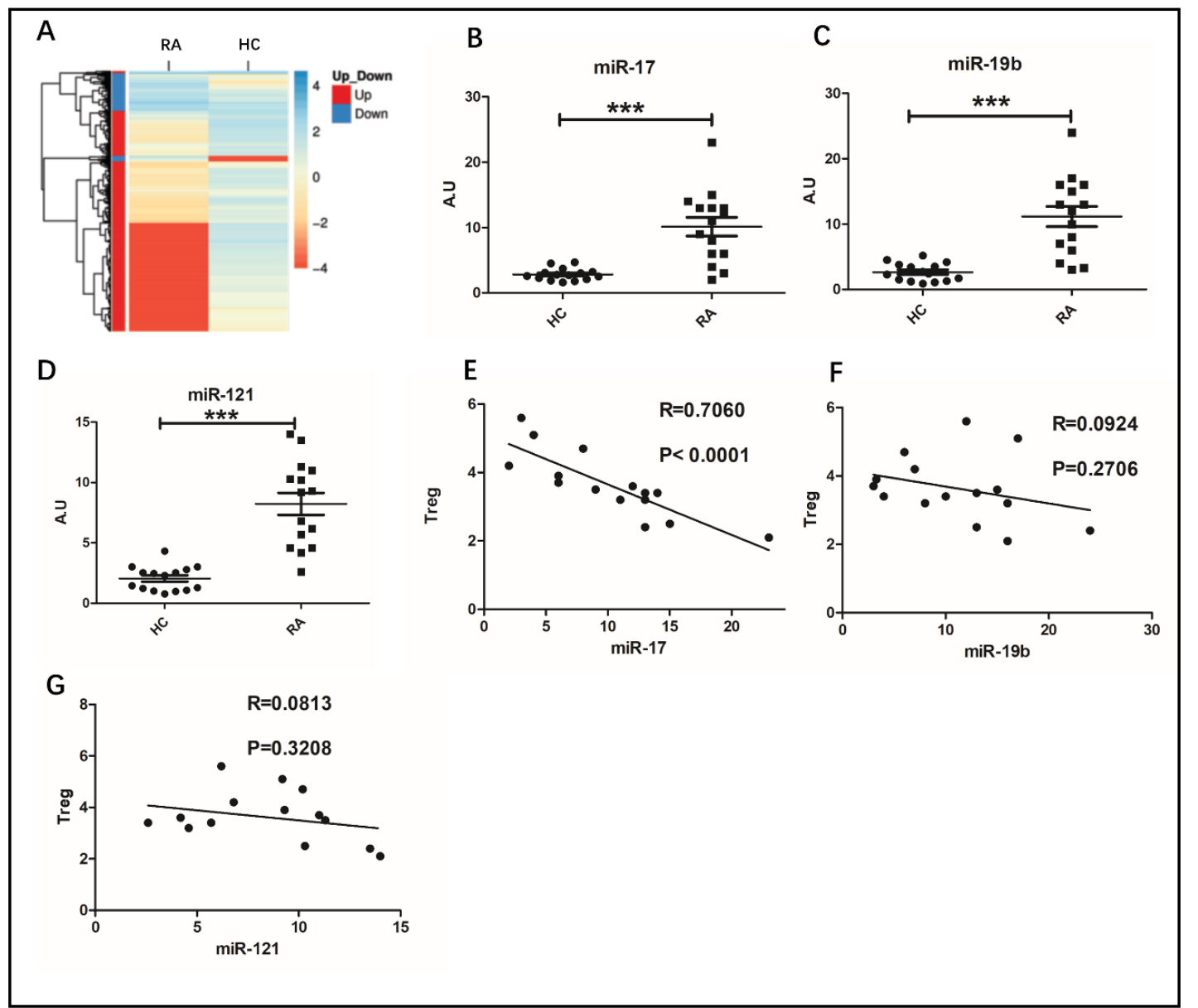

Fig. 2. Exosomal miR-17 is increased and inversely correlates with Treg frequency in RA patients. A, Heat map of miRNA expression profile in the exosomes from HC and RA patients. RNA was extracted from exosomes, which were isolated from the plasma of one HC and one RA patient. B-D, Quantification of the candidate miRNAs by RT-qPCR. The total amount of RNAs in the exosomes from the same amount of plasma was also examined. E-G, Correlation between the frequency of Tregs and exosomal miRNAs in patients with RA. The data indicate the mean \pm SEM of 15 subjects.

Given that Treg frequency was decreased in RA patients, we focused on the effect of exosomes on Tregs. We cultured peripheral blood CD4 ${ }^{+} \mathrm{T}$ cells from HCs with anti-CD3 mAb and anti-CD28 mAb in the presence of RA-exosomes or HC-exosomes. Interestingly, RAexosomes resulted in a decreased ratio of Tregs compared with HC-exosomes (Fig. 1E and F).

Exosomal miR-17 is increased and inversely correlates with Treg frequency in RA patients

To determine how RA-exosomes affect Tregs, we further analyzed miRNA expression profiles in RA-exosomes as compared with those in HC-exosomes. Microarray analysis results showed remarkable differences in miRNA profiles between RA-exosomes and HCexosomes (Fig. 2A). Next, we selected three miRNAs, namely, miR-17, miR-19b, and miR-121, for further analysis, the expression of which showed significant differences between RAexosomes and HC-exosomes (Fig. 2B-D).

We investigated whether a relationship exists between the frequency of the Tregs and these three miRNAs (miR-17, miR-19b, and miR-121) in RA patients. A negative correlation between miR-17 and Treg frequency was observed, but no correlation between the Tregs and either of the remaining two miRNAs was observed (Fig. 2E-G). 


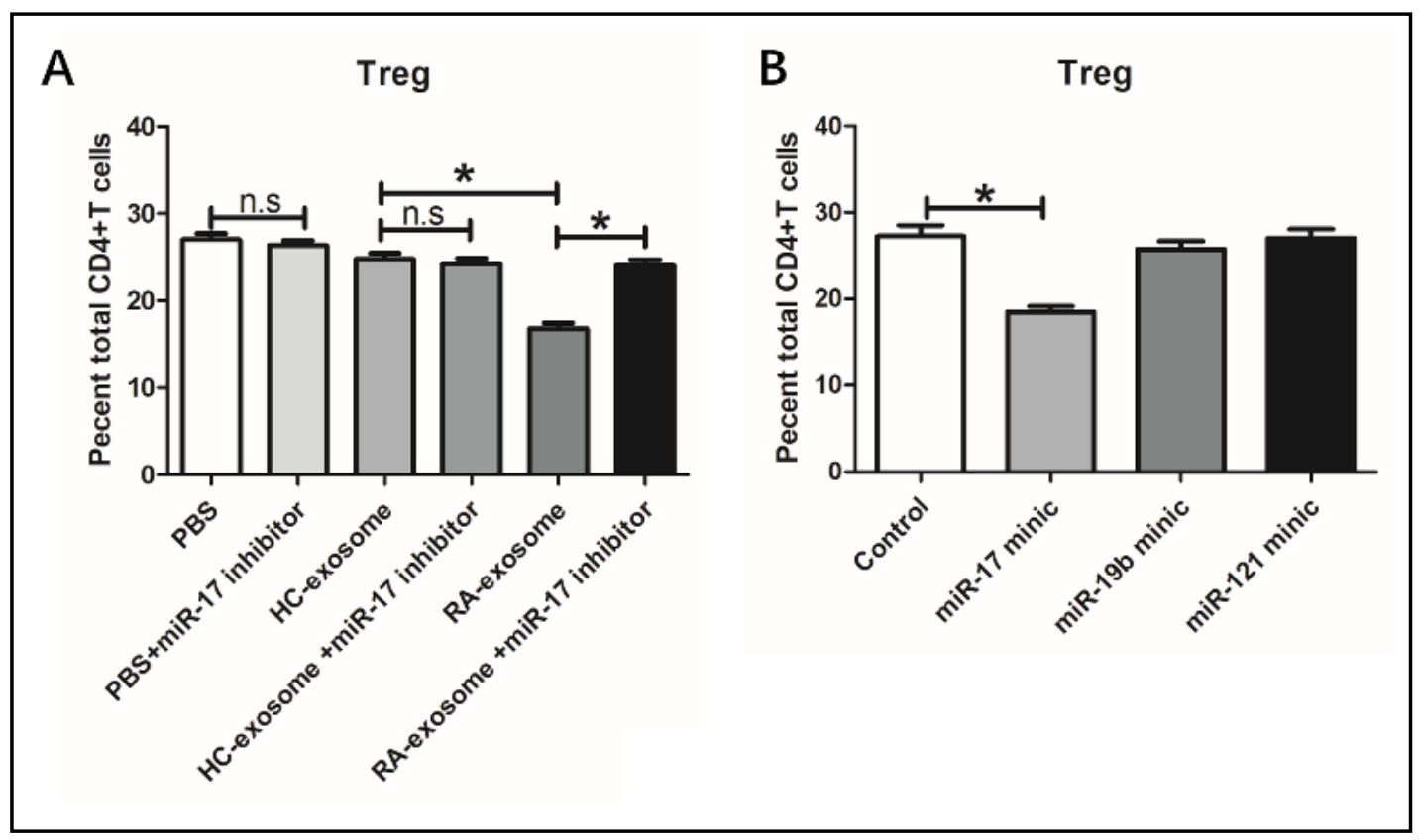

Fig. 3. RA-exosomes inhibit the differentiation of Tregs via miR-17. A, CD4+ ${ }^{+}$cells were cultured in media supplemented with PBS, HC-exosomes, or RA-exosomes, with or without miR-17 inhibitor. The data indicate the mean \pm SEM of three independent experiments. B, CD4+ T cells were transfected with miR-17, miR-19b, or miR-121 mimic. The data are representative of three independent experiments.

\section{RA-exosomes inhibit the differentiation of Tregs by miR-17}

To investigate the effect of miR-17, miR-19b, and miR-121 on Treg differentiation, $\mathrm{CD}^{+} \mathrm{T}$ cells from HCs were transfected with miR-17, miR-19b, or miR-121 mimic, cultured with anti-CD3 and anti-CD28 mAbs for $72 \mathrm{~h}$, and then the frequencies of Treg populations were evaluated by flow cytometry. The frequency of Tregs was significantly decreased after transfection with miR-17 mimic, while the frequency of Tregs was not affected after transfection with miR-19b ormiR-121 mimic (Fig. 3B). Importantly, miR-17 inhibitor significantly upregulated the frequency of Tregs in the RA-exosome group, and the rate of increase in Treg frequency after treatment with the miR-17 inhibitor was greater in the presence of RA-exosomes than HC-exosomes (Fig. 3A).

\section{miR-17 inhibits Treg differentiation through targeting TGFBR II}

To determine the mechanism of miR-17-mediated inhibition of Treg induction, we have attempted to identify target genes of miR-17. Using an online database for target prediction, Target Scan Human, we were able to identify candidate genes targeted by miR-17. Given that TGF- $\beta$ signaling pathways promote induction of Tregs, we evaluated the effect of miR-17 on target gene TGFBR II expressed by T cells. CD4 ${ }^{+} \mathrm{T}$ cells were transfected with miR-17 and cultured in the presence of anti-CD3 and anti-CD28 mAbs for $72 \mathrm{~h}$. Notably, the expression of TGFBR II on the cultured CD4+ $\mathrm{T}$ cells was significantly decreased (Fig. 4A).

To investigate whether miR-17 regulates Treg differentiation via the TGFBR II $3^{\prime}$ untranslated region (3'-UTR), we performed a luciferase reporter assay. HEK 293 T cells were transiently transfected with these constructs and miR-17 mimic or NC. miR-17 mimic significantly suppressed the luciferase activity of reporter genes containing the wild-type $3^{\prime}$ UTR of TGFBR II (Fig. 4B). In contrast, the inhibition was fully rescued when all target sites were mutated (Fig. 4B). 


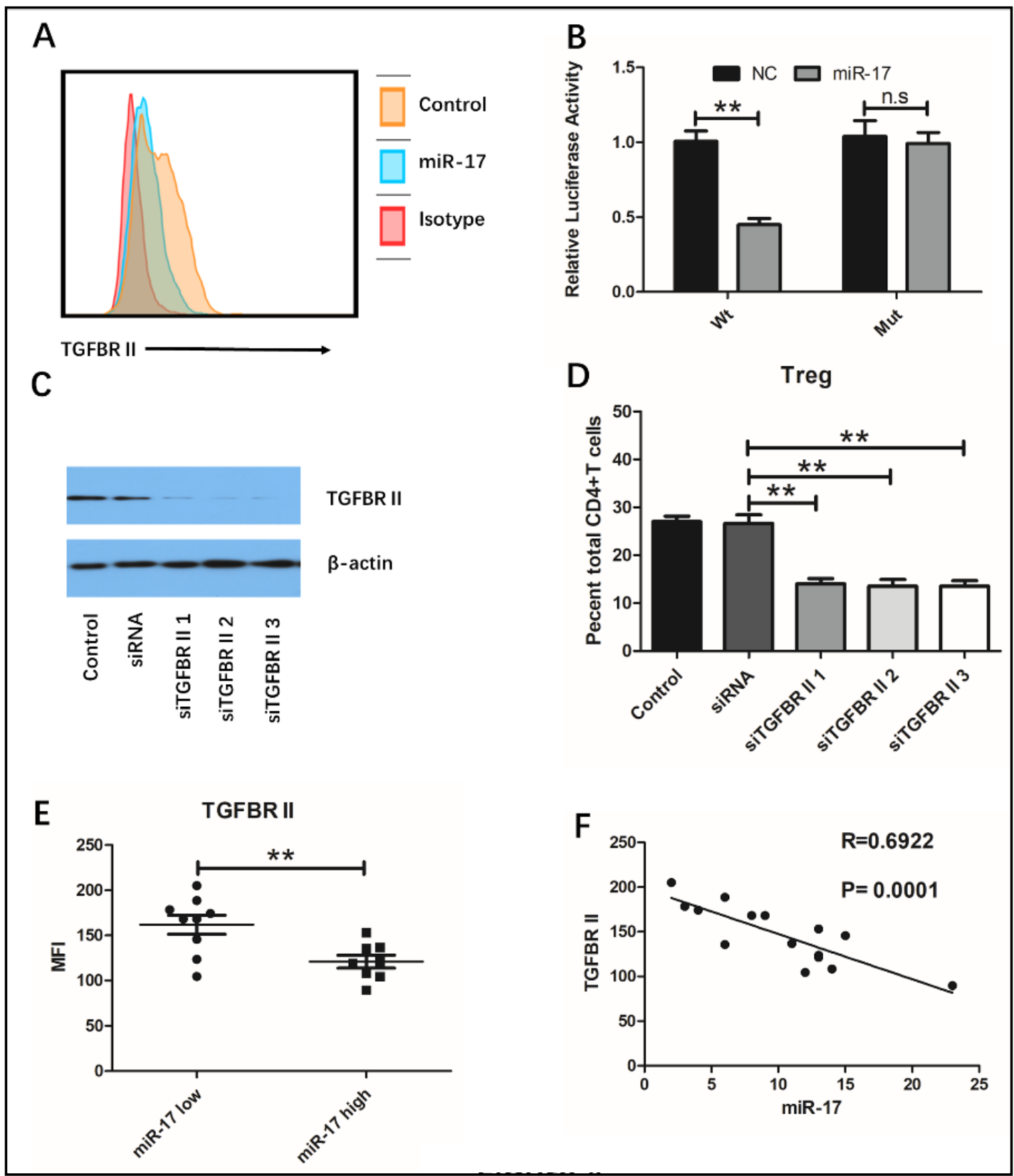

Fig. 4. miR-17 inhibits Treg differentiation through targeting TGFBR II. A, CD4+ ${ }^{+}$cells were transfected with miR-17 and then cultured under stimulation with anti-CD3 and anti-CD28 mAbs for $72 \mathrm{~h}$. Representative histograms of the expression levels of TGFBR II are shown. B, Luciferase activity of the wild-type TGFBR II 3'-UTR reporter vectors was decreased by overexpression of miR-17 in HEK 293 cells, while it was not significantly decreased when all binding sites were mutated compared with the negative control. C, Western blot analysis of $\mathrm{CD} 4^{+} \mathrm{T}$ cells transfected with either siRNA against TGFBR II (siTGFBR II-1 and -2) or NCsiRNA (siRNA). The data shown are representative of three independent experiments. $\mathrm{D}, \mathrm{CD} 4^{+} \mathrm{T}$ cells were transfected with two siRNAs targeting TGFBR II and cultured under stimulation with anti-CD3 and antiCD28 mAbs for $72 \mathrm{~h}$. The data are representative of three independent experiments. E, The frequency of Tregs and the expression of TGFBR II on CD4 ${ }^{+} \mathrm{T}$ cells were compared between the groups with lower and higher amounts of miR-17 in exosomes in the blood. F, Correlation analysis between the amount of miR17 in CD4 ${ }^{+} \mathrm{T}$ cells and the expression of TGFBR II on $\mathrm{CD} 4^{+} \mathrm{T}$ cells in RA patients. MFI, mean fluorescence intensity. 
To evaluate the effect of TGFBR II on Treg differentiation, peripheral blood CD $4^{+}$T cells were transfected with various siRNAs and cultured in the presence of anti-CD3 and anti-CD28 mAbs for $72 \mathrm{~h}$ before flow cytometry analysis. We confirmed that TGFBR II was knocked down by the three different siRNAs (Fig. 4C), which resulted in a similar reduction in the frequency of Tregs (Fig. 4D).

To obtain supportive evidence for the alteration of the miR-17/TGFBR II axis in patients with RA in vivo, we isolated PBMCs from HCs and RA patients. Strikingly, the results showed that the expression of TGFBR II on $\mathrm{CD}^{+} \mathrm{T}$ cells was significantly lower in the miR-17-high group than in the miR-17-low group (Fig. 4E). Moreover, a significant negative correlation between them was also detected (Fig. 4F).

\section{Discussion}

In the present study, we describe a novel effect of exosomes on Tregs in RA patients: RAexosomes can suppress Treg induction by the transfer of miR-17, and then miR-17 inhibits the differentiation of Tregs by targeting the expression of TGFBR II.

Tregs have been identified as sentinels of the immune response, keeping aberrant immune reactions in check and preventing autoimmunity. We and other groups have shown that a reduction of Treg frequency is an immunological hallmark of RA [3, 4, 17]. Because exosomes were shown to contribute to intercellular communication by transporting signals into the target cells either close to or distant from the cells of exosome origin $[12,16]$, we investigated the effect of RA-exosomes on Treg differentiation and found that RA-exosomes could inhibit Treg differentiation in vitro. Because miRNA has been shown to play an important role in the differentiation and function of immune cells [18-23], we next analyzed miRNA expression profiles in RA-exosomes as compared with those in HC-exosomes by microarray analysis. The results showed remarkable differences in miRNA profiles between RA-exosomes and HC-exosomes. Moreover, a negative correlation between miR-17 and Treg frequency was observed. The frequency of Tregs was significantly decreased after transfection with miR-17, and miR-17 inhibitor significantly upregulated the frequency of Tregs in the RA-exosome group. Together, these results suggest that RA-exosomes prevent the differentiation of Tregs through transporting miR-17 into T cells.

miRNAs are short noncoding RNAs with a length of 18-22 nucleotides that cause mRNA cleavage and subsequent degradation by binding to the complementary $3^{\prime}$-UTR of the mRNA. miR-17 has been shown to control iNKT cell oncogenes is via modulation of TGF- $\beta$ signaling [24], and TGF- $\beta$ signaling plays an important role in Treg differentiation [25]. Therefore, we evaluated the effect of miR-17 on TGFBR II expressed by T cells and found that the expression of TGFBR II on the cultured CD4 $4^{+} \mathrm{T}$ cells was significantly decreased after transfecting with miR-17, and siTGFBR II resulted in a similar reduction in the frequency of Tregs, suggesting that miR-17 regulated the Treg differentiation by inhibiting TGFBR II expression. Moreover, luciferase reporter assay results showed that the miR-17 mimic significantly suppressed the luciferase activity of reporter genes that contain the wild-type $3^{\prime}$-UTR of TGFBR II and the inhibition was fully rescued when all target sites were mutated, which suggests that TGFBR II is the target gene of miR-17 and miR-17 inhibits the Treg differentiation by targeting TGFBR II expression.

In this study, we found that RA-exosomes can selectively affect Tregs in vitro. Several miRNAs are more abundant in RA-exosomes than in HC-exosomes. Among those upregulated in patients with RA, miR-17 can suppress Treg induction by inhibiting the expression of TGFBR II. Our findings imply that altered miRNA expression in RA-exosomes may contribute to the pathogenesis of RA by disrupting the homeostasis of Tregs. 


\section{Cellular Physiology Cell Physiol Biochem 2018;50:1754-1763 \begin{tabular}{ll|l|l|l} 
DOl: 10.1159/000494793 & 2018 The Author(s). Published by S. Karger AG, Basel \\
and Biochemistry & $\begin{array}{l}\text { Published online: } 2 \text { November } 2018 \\
\text { www.karger.com/cpb }\end{array}$
\end{tabular}}

Wang et al.: Exosomal miR-17 Inhibits Treg Cells via Targeting TGFBR II

\section{Conclusion}

Our findings imply that altered miRNA expression in RA-exosomes may contribute to the pathogenesis of RA by disrupting the homeostasis of Tregs.

\section{Acknowledgements}

This work was supported by Gansu Health Industry Scientific Research Project (GSWSKY2016-39).

\section{Disclosure Statement}

The authors declare no conflicts of interest.

\section{References}

1 Fessler J, Felber A, Duftner C, Dejaco C: Therapeutic potential of regulatory T cells in autoimmune disorders. BioDrugs 2013;27:281-291.

2 Lawson CA, Brown AK, Bejarano V, Douglas SH, Burgoyne CH, Greenstein AS, Boylston AW, Emery P, Ponchel F, Isaacs JD: Early rheumatoid arthritis is associated with a deficit in the CD4+CD25 high regulatory T cell population in peripheral blood. Rheumatology 2006;45:1210-1217.

- Ehrenstein MR, Evans JG, Singh A, Moore S, Warnes G, Isenberg DA, Mauri C: Compromised function of regulatory T cells in rheumatoid arthritis and reversal by anti-TNFalpha therapy. J Exp Med 2004;200:277285.

-4 Nie H, Zheng Y, Li R, Guo TB, He D, Fang L, Liu X, Xiao L, Chen X, Wan B, Chin YE, Zhang JZ: Phosphorylation of FOXP3 controls regulatory T cell function and is inhibited by TNF-alpha in rheumatoid arthritis. Nat Med 2013;19:322-328.

5 Bartel DP: MicroRNAs: genomics, biogenesis, mechanism, and function. Cell 2004;116:281-297.

6 Bartel DP: MicroRNAs: target recognition and regulatory functions. Cell 2009;136:215-233.

7 Shukla GC, Singh J, Barik S: MicroRNAs: Processing, Maturation, Target Recognition and Regulatory Functions. Mol Cell Pharmacol 2011;3:83-92.

8 Simpson LJ, Ansel KM: MicroRNA regulation of lymphocyte tolerance and autoimmunity. J Clin Invest 2015;125:2242-2249.

-9 Sode J, Krintel SB, Carlsen AL, Hetland ML, Johansen JS, Horslev-Petersen K, Stengaard-Pedersen K, Ellingsen T, Burton M, Junker P, Ostergaard M, Heegaard NHH: Plasma MicroRNA Profiles in Patients with Early Rheumatoid Arthritis Responding to Adalimumab plus Methotrexate vs Methotrexate Alone: A Placebo-controlled Clinical Trial. J Rheumatol 2018;45:53-61.

-10 Mohammadnia-Afrouzi M, Hosseini AZ, Khalili A, Abediankenari S, Amari A, Aghili B, Nataj HH: Altered microRNA Expression and Immunosuppressive Cytokine Production by Regulatory T Cells of Ulcerative Colitis Patients. Immunol Invest 2016;45:63-74.

11 Soltanzadeh-Yamchi M, Shahbazi M, Aslani S, Mohammadnia-Afrouzi M: MicroRNA signature of regulatory T cells in health and autoimmunity. Biomed Pharmacother 2018;100:316-323.

12 Valadi H, Ekstrom K, Bossios A, Sjostrand M, Lee JJ, Lotvall JO: Exosome-mediated transfer of mRNAs and microRNAs is a novel mechanism of genetic exchange between cells. Nat Cell Biol 2007;9:654-659.

13 Kosaka N, Iguchi H, Yoshioka Y, Takeshita F, Matsuki Y, Ochiya T: Secretory mechanisms and intercellular transfer of microRNAs in living cells. J Biol Chem 2010;285:17442-17452.

14 Tkach M, Thery C: Communication by Extracellular Vesicles: Where We Are and Where We Need to Go. Cell 2016;164:1226-1232.

15 Becker A, Thakur BK, Weiss JM, Kim HS, Peinado H, Lyden D: Extracellular Vesicles in Cancer: Cell-to-Cell Mediators of Metastasis. Cancer cell 2016;30:836-848. 


\section{Cellular Physiology Cell Physiol Biochem 2018;50:1754-1763

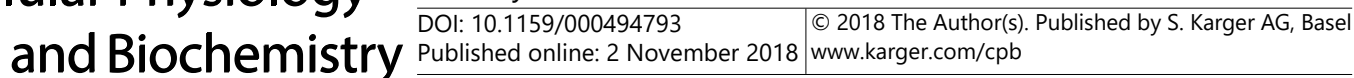

Wang et al.: Exosomal miR-17 Inhibits Treg Cells via Targeting TGFBR II

-16 Zappulli V, Friis KP, Fitzpatrick Z, Maguire CA, Breakefield XO: Extracellular vesicles and intercellular communication within the nervous system. J Clin Invest 2016;126:1198-1207.

17 Fessler J, Raicht A, Husic R, Ficjan A, Schwarz C, Duftner C, Schwinger W, Graninger WB, Stradner MH, Dejaco C: Novel Senescent Regulatory T-Cell Subset with Impaired Suppressive Function in Rheumatoid Arthritis. Front Immunol 2017;8:300.

18 Chen S, Zeiser R: The Role of MicroRNAs in Myeloid Cells during Graft-versus-Host Disease. Front Immunol 2018;9:4.

19 Baulina N, Kulakova O, Kiselev I, Osmak G, Popova E, Boyko A, Favorova O: Immune-related miRNA expression patterns in peripheral blood mononuclear cells differ in multiple sclerosis relapse and remission. J Neuroimmunol 2018;317:67-76.

20 Zhang Y, Liu W, Chen Y, Liu J, Wu K, Su L, Zhang W, Jiang Y, Zhang X, Zhang Y, Liu C, Tao L, Liu B, Zhang H: A Cellular MicroRNA Facilitates Regulatory T Lymphocyte Development by Targeting the FOXP3 Promoter TATA-Box Motif. J Immunol 2018;200:1053-1063.

21 Ji X, Wang E, Tian F: MicroRNA-140 suppresses osteosarcoma tumor growth by enhancing anti-tumor immune response and blocking mTOR signaling. Biochem Biophys Res Commun 2018;495:1342-1348.

22 Botta C, Cuce M, Pitari MR, Caracciolo D, Gulla A, Morelli E, Riillo C, Biamonte L, Gallo Cantafio ME, Prabhala R, Mignogna C, Di Vito A, Altomare E, Amodio N, Di Martino MT, Correale P, Rossi M, Giordano A, Munshi NC et al.: MiR-29b antagonizes the pro-inflammatory tumor-promoting activity of multiple myeloma-educated dendritic cells. Leukemia 2017.

23 Singh PB, Pua HH, Happ HC, Schneider C, von Moltke J, Locksley RM, Baumjohann D, Ansel KM: MicroRNA regulation of type 2 innate lymphoid cell homeostasis and function in allergic inflammation. J Exp Med 2017;214:3627-3643.

-24 Fedeli M, Riba M, Garcia Manteiga JM, Tian L, Vigano V, Rossetti G, Pagani M, Xiao C, Liston A, Stupka E, Cittaro D, Abrignani S, Provero P, Dellabona P, Casorati G: miR-17 approximately 92 family clusters control iNKT cell ontogenesis via modulation of TGF-beta signaling. Proc Natl Acad Sci U S A 2016;113:E8286-E8295.

25 Freudenberg K, Lindner N, Dohnke S, Garbe AI, Schallenberg S, Kretschmer K: Critical Role of TGF-beta and IL-2 Receptor Signaling in Foxp3 Induction by an Inhibitor of DNA Methylation. Front Immunol 2018;9:125. 